\section{Antimicrobial resistance: a major priority for global focus}

\author{
Jonathan Cooke
}

This special edition is dedicated to antimicrobial resistance (AMR) which has become a highly significant concern for governments and healthcare providers globally.

AMR threatens the effective prevention and treatment of an ever-increasing range of infections caused by bacteria, parasites, viruses and fungi, and many countries have adopted strategies to address AMR. ${ }^{1}$

There were an estimated 4.95 million (3.62-6.57) deaths associated with bacterial AMR in $2019,{ }^{2}$ and AMR is predicted to cause 10 million global deaths annually by 2050 at a cost of US\$100 trillion. ${ }^{3}$ The recent SARS-CoV-2 (COVID-19) pandemic has, to date, caused 5.7 million global deaths since the first cases were identified.

Putting AMR in clinical context, a hospital study found between $38.7 \%$ and $50.9 \%$ of pathogens causing surgical site infections and $26.8 \%$ of pathogens causing infections after chemotherapy were already resistant to standard prophylactic antibiotics in the USA. ${ }^{4}$ This has worrying implications for patients attending for surgery and cancer in the future.

There are two main types of AMR: inherent and acquired.

SARS-CoV-2 (COVID-19) is a good example of inherent resistance as a viral pathogen emerging with little susceptibility to existing antiviral agents.

Acquired resistance invariably has developed as a response to the use and overuse of antimicrobials in clinical medicine and farming. Common examples include methicillin-resistant Staphylococcus aureus, vancomycin-resistant enterococci and Clostridium difficile.

In its Global Action Plan on AMR, WHO outlines five key objectives ${ }^{5}$ :

1. To improve awareness and understanding of AMR through effective communication, education and training.

2. To strengthen the knowledge and evidence base through surveillance and research.

Correspondence to Professor Jonathan Cooke, Manchester Pharmacy School, The University of Manchester, Manchester, UK; jonathan.cooke@ manchester.ac.uk
3. To reduce the incidence of infection through effective sanitation, hygiene and infection prevention measures.

4. To optimise the use of antimicrobial medicines in human and animal health.

5. To develop the economic case for sustainable investment that takes account of the needs of all countries and to increase investment in new medicines, diagnostic tools, vaccines and other interventions.

With the current SARS-CoV-2 (COVID19) pandemic, it is likely that the inappropriate use of antimicrobials has become more prevalent so urgent action is needed to address this.

The papers that are published in this special edition cover a wide range of topics from authors worldwide. The following topics are covered with their respective alignment, as (n), to the $\mathrm{WHO}$ AMR objectives above.

András Süle, President of the European Association of Hospital Pharmacists (EAHP), reviews the importance of the pharmacists' involvement in frontline patient care within hospitals, in general, and in AMR, in particular (1); Kousovista et al describe a time series analysis of colistin-resistant Acinetobacter baumannii strains (2); Powell et al examine the opportunity to reduce the length of courses of antibiotics in the emergency department in line with national recommendations (4); Heireman et al describe the effects of cascade reporting of susceptibility profiles for Enterobacterales on broadspectrum antibiotics use and resistance (2); Veličković-Radovanović et al review a 5-year surveillance of antimicrobial use and multidrug-resistant (MDR) isolates of Escherichia coli and Klebsiella spp MDR isolates in a tertiary healthcare centre (2); using lessons from EAHP and the European Centre for Disease Prevention and Control (ECDC) surveys, Miljković et al identify the management of antibiotic shortages as a contributing factor for AMR stewardship (4); an up-to-date review of resistance in fungal infections is provided by Denning (1); Bejarano et al describe an individual case report of the successful treatment of Verona integronencoded metallo- $\beta$-lactamase-producing
Klebsiella pneumoniae infection using the combination of ceftazidime/avibactam and aztreonam (4); and finally, Iheanacho and Eze describe particular problems with high antimicrobial use but low AMR awareness in Nigeria (2).

A fundamental aspect of clinical therapeutics is that effective treatment can only be instigated when there is diagnostic certainty. One aspect of AMR strategies that need to be addressed more is the importance of accurate diagnosis of the presence of an infection. Genomic assays have brought high levels of sophistication in the determination of genetic aspects of microorganisms that cause infections with high accuracy in predicting the optimal selection of antimicrobial agents. However, there is considerable variation in the ability to obtain an effective sample in cases of certain clinical infectious diseases in order to do genomic testing. In certain case, this can be in up to half of patients presenting with symptoms of an infection. ${ }^{6}$ Thus, tests that measure the systemic effects of the presence of an infection as well as microbiological and genomic testing are crucial to improve clinical decision-making.

These tests should be undertaken in conjunction with clinical examination and with diagnostic tests on clinical, physical, biochemical and biomarker parameters associated with infectious diseases. Pointof-care (POC) biomarker tests are now being advocated by national governments, alongside antimicrobial stewardship, as a means of adding precision to diagnosis and management of infectious diseases in patients presenting to clinics and community pharmacies. ${ }^{7}$ In particular, $\mathrm{C}$ reactive protein POCT has been shown to reduce antibiotic prescribing for respiratory tract infections by at least $25 \% .^{89}$

AMR continues to be a global challenge to health and focus must be maintained in ensuring that antimicrobials are used optimally in all healthcare settings.

Funding The author has not declared a specific grant for this research from any funding agency in the public, commercial or not-for-profit sectors.

Competing interests Professor Cooke chaired the Antimicrobial Stewardship Subgroup of the Department of Health Advisory Committee on Antimicrobial Resistance and Healthcare Associated Infection (travel expenses only), and has chaired, presented and received honoraria at meetings supported by Astellas, Cubist, Alere and $\mathrm{HHI}$.

Provenance and peer review Commissioned; internally peer reviewed.

(C) European Association of Hospital Pharmacists 2022. No commercial re-use. See rights and permissions. Published by BMJ. 


\section{Check for updates}

To cite Cooke J. Eur J Hosp Pharm 2022;29:63-64.

Eur J Hosp Pharm 2022;29:63-64.

doi:10.1136/ejhpharm-2022-003241

\section{ORCID iD}

Jonathan Cooke http://orcid.org/0000-0001-50169048

\section{REFERENCES}

1 European Commission. A European One-Health action plan against antimicrobial resistance (AMR) 2017.

2 Antimicrobial Resistance Collaborators. Global burden of bacterial antimicrobial resistance in 2019: a systematic analysis. Lancet 2022. doi:10.1016/\$01406736(21)02724-0. [Epub ahead of print: 18 Jan 2022].

3 O'Neill J. Antimicrobial resistance: tackling a crisis for the health and wealth of nations. London: HM Government and Wellcome Trust, 2014.

4 Teillant A, Gandra S, Barter D, et al. Potential burden of antibiotic resistance on surgery and cancer chemotherapy antibiotic prophylaxis in the USA: a literature review and modelling study. Lancet Infect Dis 2015;15:1429-37.

5 World Health Organisation (WHO). Global action plan on antimicrobial resistance 2015.

6 Baron EJ, Miller JM, Weinstein MP, et al. Executive summary: a guide to utilization of the microbiology laboratory for diagnosis of infectious diseases: 2013 recommendations by the Infectious Diseases Society of America (IDSA) and the American
Society for Microbiology (ASM)(a). Clin Infect Dis 2013;57:485-8.

7 NHS England and NHS Improvement. Point of care testing in community pharmacies Guidance for commissioners and community pharmacies delivering NHS services. In: NHS England and NHS improvement, ed. London: NHS England, 2022.

8 Verbakel JY, Lee JJ, Goyder C, et al. Impact of point-of-care $C$ reactive protein in ambulatory care: a systematic review and meta-analysis. BMJ Open 2019;9:1-11.

9 Cooke J, Llor C, Hopstaken R, et al. Respiratory tract infections (RTIs) in primary care: narrative review of $C$ reactive protein (CRP) point-of-care testing (POCT) and antibacterial use in patients who present with symptoms of RTI. BMJ Open Respir Res 2020;7:e000624. 